\title{
Angiomyofibroblastoma of Vulva. A Rare Tumor Entity: A Case Report
}

\author{
Mohammad Shahid Iqbal ${ }^{1}$ M.D, Unmesh Santpur ${ }^{2}$ M.D, Aisha Tabassum ${ }^{3}$ M.D, \\ Kotha Vamshi Krishna ${ }^{4}$ M.D,V.Satyanarayana ${ }^{5}$ M.D . \\ ${ }^{I}$.Assistant professor,Department of Pathology, kamineni institute of medical sciences,Narketpally,A.P \\ ${ }^{2}$. Associate professor,Department of $O B G$, kamineni institute of medical sciences, Narketpally,A.P \\ ${ }^{3}$ Assistant professor,Department of Pathology, kamineni institute of medical sciences ,Narketpally,A.P \\ ${ }^{4}$.Assistant professor,Department of Radiology, kamineni institute of medical sciences, Narketpally,A.P \\ 5. Professor and Head,Department of Pathology, kamineni institute of medical sciences, Narketpally,A.P
}

\begin{abstract}
Angiomyofibroblastoma is a rare,usually small benign mesenchymal tumor that occurs mainly but not exclusively in the vulval region of premenopausal women. Patients often present with non specific symptoms. It is a well circumscribed lesion that clinically is often thought to represent a Bartholin's gland cyst. Diagnostic confusion often arise between angiomyofibroblastoma and other tumors arising in the vulval region like aggressive angiomyxoma and cellular angiofibroma because of overlapping histological features. Differentiating between angiomyofibroblastoma and cellular angiofibroma is not of clinical significance.Aggressive angiomyxoma is a more infiltrative lesion that has a high propensity for local recurrence. Immunohistochemistry is not of much help in distinguishing these entities. Sincere efforts should be made to differentiate angiomyofibroblastoma from aggressive angiomyxoma, which has a potential to metastasize or recur.
\end{abstract}

Key words: Angiomyofibroblastoma, Vulva, aggressive angiomyxoma

Submitted Date 22 June 2013

Accepted Date: 27 June 2013

\section{Introduction}

Angiomyofibroblastoma is a rare benign mesenchymal tumor usually occurring in the vulvovaginal area of middle aged women ${ }^{1 .}$ Fletcher and colleagues first described angiomyofibroblastoma in 1992. The importance of this rare neoplasm is related to its potential mimicry of more infiltrative and prognostically less favourable lesions such as aggressive angiomyxomas ${ }^{2}$. Aggressive angiomyxoma is a more infiltrative neoplasm that has a high propensity for local recurrence in contrast to an angiomyofibroblastoma ${ }^{3}$.Angiomyofibroblastoma is known to have distinct clinicopathologic features as a non aggressive benign mesenchymal tumor. The histology of angiomyofibroblastoma is characterized by bland looking stromal cells and abundant small blood vesssels. ${ }^{4}$

We report a case of angiomyofibroblastoma along with review of literature.

\section{Case details}

A 45 year old female patient came to the gynecological out patient clinic with a 03 month history of painless swelling in the right labia majora. Physical examination of the swelling revealed a soft to firm,mobile mass measuring approximately $08 \mathrm{~cm}$ in largest dimension. Clinically,labial cyst was the diagnosis. Laboratory data were unremarkable. On radiological imaging, a provisional diagnosis of ischiorectal angiomyxoma was made keeping in mind differential diagnosis of angiomyofibroblastoma and other mesenchymal tumors. The lesion was removed by simple excision. Grossly,the lesion was well circumscribed measuring $9 \mathrm{~cm}$ in the greatest dimension. Cut surface was gray white with occasional areas of myxoid appearance. Histologically, tumor showed alternating hypocellular and hypercellular areas comprising of many small to medium sized blood vessels. Blood vessels were predominantly thin walled capillary type. Occasionally very few blood vessels were of large size and showed thick hyalinized walls. Stroma consisted of plump spindle and ovoid cells scattered and occasionally forming aggregates around blood vessels. A few loose myxoid areas were present. No mucin was present. No cellular features of malignancy were present. No foci of hemorrhage was seen. Sparsely scattered adipocytes were seen within the stroma. Based on the above histological features, the diagnosis made was angiomyofibroblastoma of vulva. 


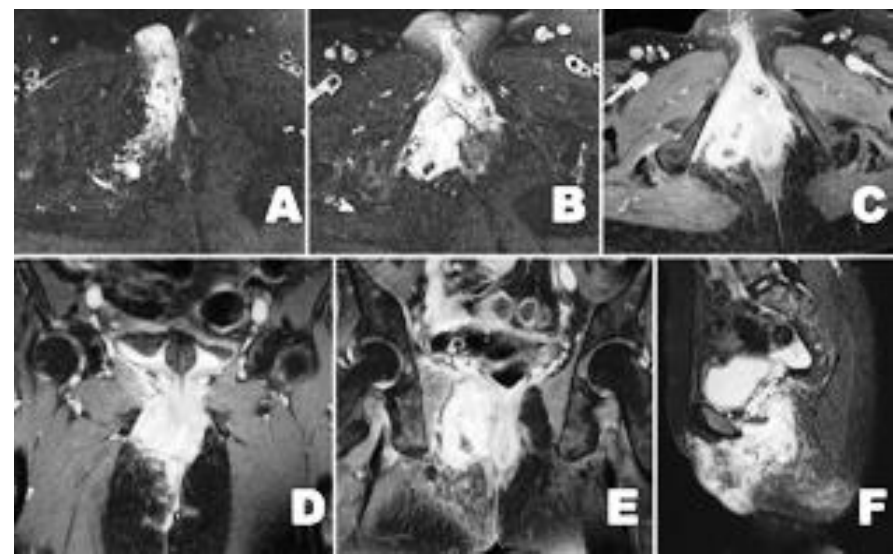

Fig.1.A and B) T2fs axial images showing lesion arising from the right vaginal wall extending into the right ischiorectal fossa C) Post contrast T1fs axial image showing early heterogenous enhancement of mass $\mathrm{D}$ and $\mathrm{E}$ ) Post contrast T1fs coronal images showing enhancing mass and its relation with the vagina and anus F) Post contrast T1fs image showing the full superoinferior extent of mass

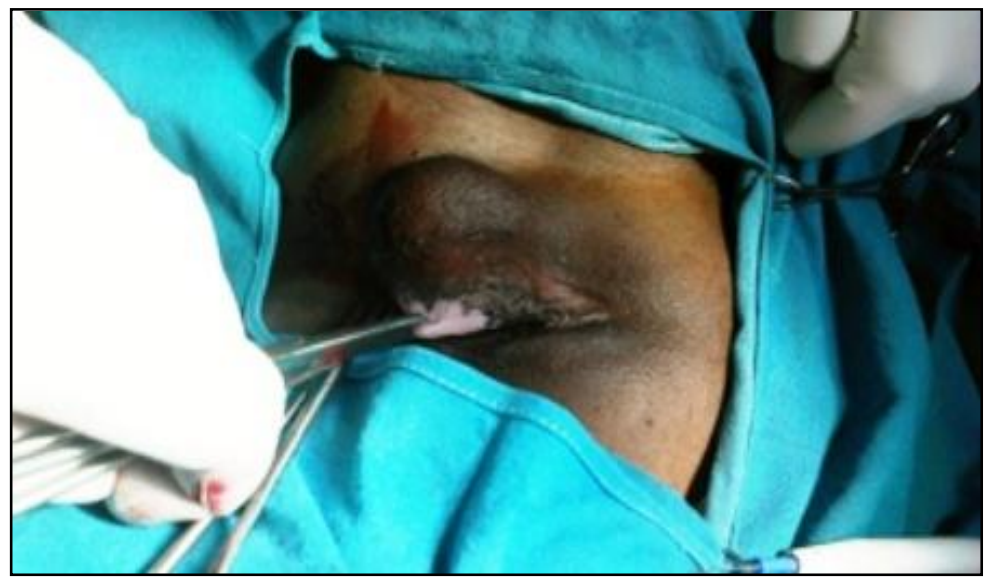

Fig.2: Swelling arising in the right labia majora

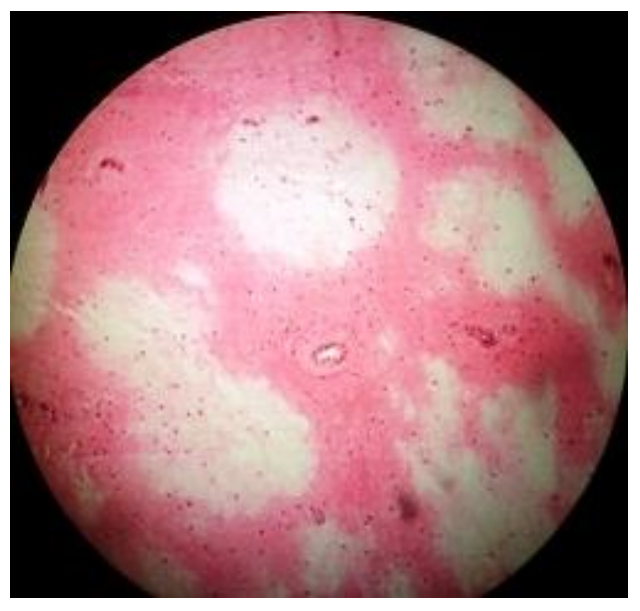

Fig.3: Alternating hypocellular and hypercellular edematous areas

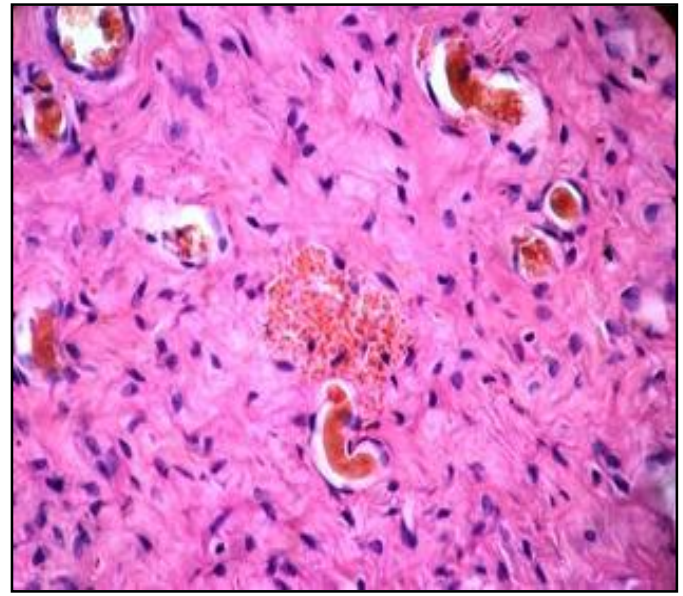

Fig.4: Capillary type blood vessels with spindle and plump stromal cells 


\section{Discussion}

Angiomyofibroblastoma is a rare mesenchymal tumor found in the vulva. It usually presents as a non tender vulval swelling ${ }^{5}$. Angiomyofibroblastoma occurs mainly,but not exclusively in the vulval region of premenopausal women ${ }^{6}$. Most cases of angiomyofibroblastoma measures less than $5 \mathrm{~cm}$. In literature, the previously reported cases ranged from $0.5 \mathrm{~cm}$ to $13 \mathrm{~cm}$ in their greatest dimension ${ }^{4}$. Histologically, angiomyofibroblastoma is a well demarcated tumor and well vascularized with alternating hypocellular and hypercellular edematous areas composed of bland,plump,spindle or oval to spindle stromal cells,frequently aggregated around blood vessels ${ }^{7}$.

Preoperative differential diagnosis includes bartholin gland cyst,labial cyst,inguinal hernia and mesenchymal tumors such as lipoma,angiomyofibroblastoma and aggressive angiomyxoma ${ }^{4}$. The diagnosis of angiomyofibroblastoma is complicated because of its shared histological features with other soft tissue tumors that can develop in female genital tract ${ }^{4}$. Within the vulva the chief differential diagnosis of angiomyofibroblastoma is likely to be aggressive angiomyxoma and cellular angiofibroma ${ }^{6}$.

Angiomyofibroblastoma can be distinguished from aggressive angiomyxoma by its circumscribed borders,much higher cellularity,more numerous blood vessels with lack of vessel wall hyalinization,frequent presence of plump stromal cells,minimal stromal mucin and rarity of erythrocyte extravasation ${ }^{8}$.

Angiomyofibroblastoma of the vulva is almost always a benign lesion which unlike aggressive angiomyxoma shows little or no tendency for local recurrence. However,a single case with sarcomatous transformation has been described ${ }^{6}$. Diagnostic confusion can also arise between angiomyofibroblastoma and cellular angiofibroma,both of which develop in the female genital tract and have prominent vascular components and bland stromal cells,although it is probably not of clinical significance ${ }^{4}$. In addition,both can have adipocytes or lipomatous component. The presence of round tumor cells clustered around blood vessels and a lack of numerous thick walled,hyalinized vessels and wispy collagen bundles helps distinguish angiomyofibrobllastoma from cellular angiofibroma.Immunohistochemistry do not distinguishes between angiomyofibroblastoma and aggressive angiomyxoma as immunohistochemistry profiles indicate a strong reactivity for vimentin with various expressions of desmin and alpha smooth muscle actin ${ }^{4}$.

Angiomyofibroblastoma cases are desmin positive and alpha smooth muscle actin negative. However,some are negative for desmin or positive for alpha smooth muscle actin ${ }^{6,8,9,10}$. The tumor cells are uniformly negative for cytokeratin and S-100 protein and CD34. Not all tumor cells are negative for alpha smooth muscle actin and CD34. Staining for ER and PR was observed in the nucleus of all tumor cells $1,6,8,9,10$.

Bigotti et ${ }^{11}$ al described about strong CD44 expression by aggressive angiomyxoma where as angiomyofibroblastoma shows a weak and heterogenous positivity for CD44. This might explain the more aggressive nature of aggressive angiomyxoma because this receptor is able to mediate migration of neoplastic cells on a hyaluronate rich extracellular matrix. It is speculated that the neoplastic cell of angiomyofibroblastoma and aggressive angiomyxoma of the vulva is a specific myofibroblast which probably arises from undifferentiated mesenchymal cells normally occurring in the lower female genital tract and both the tumors belong to a histopathological spectrum with overlapping features ${ }^{11}$.

Angiomyofibroblastoma is a benign tumor and is generally non recurrent. Sincere efforts should be made to differentiate angiomyofibroblastoma from aggressive angiomyxoma which have a potential to metastasize and recur.

[1]. Giannella L, Costantini M, Mfuta K, Cavazza A, Cerami LB, Gardini G et al. Pedunculated angiomyofibroblastoma of the vulva: case report and review of literature. Case reports in medicine 2011; vol 1: 1-4

[2]. Lim KJ, Moon JH, Yoon DY, Cha JH, Lee IJ, Min SJ. Angiomyofibroblastoma arising from the posterior perivesical space: a case report with MR findings. Korean J RADIOL 2008;9: 382-385

[3]. Kim SW, Lee JH, Han JK, Jeon S. Angiomyofibroblastoma of the vulva. J Ultrasound Med 2009;28: 1417-1420

[4]. Omori M, Toyoda H, Hirai T, Ogino T, Okada S. Angiomyofibroblastoma of the vulva:A large pedunculated mass formation. Acta Med Okayama 2006; vol 60(4): 237-242

[5]. Hjalmer S, Svennevik MS, Fai CK, Andua KT. Angiomyofibroblastoma and aggressive angiomyxoma.Two rare tumors of the vulva. Journal of pelvic medicine and surgery 2006; vol 12(4): 225-228

[6]. McCluggage WG. Angiomyofibroblastoma of the vagina. J Clin Pathol 2000;53: 803-806

[7]. Hisoaka M, Kouho H, Aoki T, Daimaru Y, Hashimoto H. Angiomyofibroblastoma of the vulva:A clinicopathologic study of seven cases. Pathology International 1995; vol 45(7): 487-492

[8]. Fletcher CDM, Tsang W, Fisher C, Lee KC, Chan J.Angiomyofibroblastoma of the vulva:A benign neoplasm distinct from aggressive angiomyxoma. Am J Surg Pathol 1992; vol 16(4): 364-372

[9]. Vasquez MD, Ro JY, Park YW, Tornor CS, Ordofiez NG, Ayale AG. Angiomyofibroblastoma: A clinicopathologic study of 8 cases and review of the literature. Int J Surg Pathol july 1999;vol7(3): 161-169

[10]. .Nagai K, Aadachi K, Saito H. Huge pedunculated angiomyofibroblastoma of the vulva. Int J Clin Oncol 2010;15: 201 -205

[11]. Bigotti G, Coli A, Gasbarri A, Castagnola D, Madonna V, Bartolazzi A. Pathology-Research and Practice;vol 195(1): 39-44 